\title{
openheart Prevalence and haemodynamic profiles of pulmonary hypertension in cardiac amyloidosis
}

\author{
Jeremy Slivnick (D) , ${ }^{1}$ Karolina M Zareba, ${ }^{2}$ Juliet Varghese (D) , ${ }^{2}$ Vien Truong, ${ }^{3}$ \\ Alexander L Wallner, ${ }^{2}$ Matthew S Tong, ${ }^{2}$ Christopher Hummel, ${ }^{2}$ Wojciech Mazur, ${ }^{3}$ \\ Saurabh Rajpal (1) 4,5
}

To cite: Slivnick J, Zareba KM, Varghese J, et al. Prevalence and haemodynamic profiles of pulmonary hypertension in cardiac amyloidosis. Open Heart 2022;9:e001808. doi:10.1136/ openhrt-2021-001808

Received 7 August 2021 Accepted 9 January 2022

D Check for updates

(C) Author(s) (or their employer(s)) 2022. Re-use permitted under CC BY-NC. No commercial re-use. See rights and permissions. Published by BMJ.

${ }^{1}$ Cardiovascular Medicine, University of Chicago Medicine, Chicago, Illinois, USA

${ }^{2}$ Cardiovascular Medicine, The Ohio State University Wexner Medical Center, Columbus, Ohio, USA

${ }^{3}$ Cardiology, Christ Hospital, Cincinnati, Ohio, USA ${ }^{4}$ Internal Medicine/Cardiology, Ohio State University Wexner Medical Center, Columbus, Ohio, USA

${ }^{5}$ Cardiology, Nationwide Children's Hospital Doctors Hospital Pediatric Residency Training Program, Columbus, Ohio, USA

Correspondence to Dr Jeremy Slivnick; jslivnick@ gmail.com

\section{ABSTRACT}

Objectives While cardiac amyloidosis (CA) classically involves the left ventricle (LV), less is known about its impact on the right ventricle (RV) and pulmonary vasculature. We performed a retrospective analysis to identify the prevalence and types of pulmonary hypertension $(\mathrm{PH})$ profiles in $\mathrm{CA}$ and to determine haemodynamic and cardiovascular magnetic resonance (CMR) predictors of major adverse cardiovascular events (MACE).

Methods Patients with CA who underwent CMR and right heart catheterisation $(\mathrm{RHC})$ within 1 year between 2010 and 2019 were included. Patients were assigned the following haemodynamic profiles based on RHC: no $\mathrm{PH}$, precapillary $\mathrm{PH}$, isolated postcapillary $\mathrm{PH}$ (IPCPH), or combined precapillary and postcapillary PH (CPCPH). The relationship between PH profile and MACE (death, heart failure hospitalisation) was assessed using survival analysis. CMR and RV parameters were correlated with MACE using Cox-regression analysis.

Results A total of 52 patients were included (age $69 \pm 9$ years, $85 \%$ men). RHC was performed during biopsy in $44(85 \%)$ and for clinical indications in $8(15 \%)$ patients. Rates of no PH, precapillary PH, IPCPH and CPCPH were 5 (10\%), 3 (6\%), 29 (55\%) and 15 (29\%), respectively. Haemodynamic $\mathrm{PH}$ profile did not correlate with risk of death $(p=0.98)$ or MACE $(p=0.67)$. Transpulmonary gradient (TPG) (HR 0.88, Cl 0.80 to 0.97 ), RV, (HR 0.95, Cl 0.92 to 0.98 ) and LV ejection fraction (HR $0.95, \mathrm{Cl} 0.92$ to 0.98) were significantly associated with MACE.

Conclusions $\mathrm{PH}$ is highly prevalent in $\mathrm{CA}$, even at the time of diagnosis. While IPCPH was most common, $\mathrm{CPCPH}$ is not infrequent. TPG and RV ejection fraction (RVEF) are prognostic markers in this population.

\section{INTRODUCTION}

Cardiac amyloidosis (CA) is a disorder in which abnormally folded proteins deposit in the myocardium, resulting in end-organ dysfunction. While previously thought to be rare, it is increasingly recognised as a common cause of heart failure and low-flow, low-gradient aortic stenosis in the elderly. ${ }^{12}$ CA is most commonly caused by either transthyretin (ATTR) or immunoglobulin light

\section{Key questions}

What is already known about this subject?

- Cardiac amyloidosis is an infiltrative cardiomyopathy in which abnormally folded proteins deposit in the myocardium, often resulting in restrictive cardiomyopathy and left ventricular failure. While pulmonary vascular disease has been associated with other forms of left heart failure, the prevalence and haemodynamic profiles of pulmonary hypertension in cardiac amyloidosis have not previously been explored.

What does this study add?

- In this study, we identified a high prevalence of pulmonary hypertension in patients with cardiac amyloidosis. While postcapillary pulmonary hypertension, combined pre and postcapillary pulmonary hypertension, was frequently observed.

How might this impact on clinical practice?

- Our study highlights the need for earlier detection of cardiac amyloidosis given the high prevalence of pulmonary hypertension at the time of diagnosis. Future studies are needed to assess whether therapies targeting the pulmonary vasculature can benefit appropriately phenotyped patients with this disorder.

chain (AL) protein. While the effects of CA on the left ventricle (LV) have been previously well described, less is known about its impact on the pulmonary vasculature and the right heart.

Pulmonary hypertension $(\mathrm{PH})$ is defined by societal guidelines as a mean pulmonary artery pressure $\geq 20 \mathrm{~mm} \mathrm{Hg}^{3}{ }^{3} \mathrm{PH}$ can be further subcategorised based on haemodynamic profile assessed using right heart catheterisation (RHC) into precapillary $\mathrm{PH}$, isolated postcapillary $\mathrm{PH}$ (IPCPH) or combined pre and postcapillary $\mathrm{PH}(\mathrm{CPCPH})$ (table 1). ${ }^{3}$ Previous studies have shown that these distinct $\mathrm{PH}$ haemodynamic profiles affect outcomes, with CPCPH portending the 
Table 1 Definitions of pulmonary hypertension haemodynamic profiles

\begin{tabular}{lllll}
\hline & No PH & Pre-capillary PH & $\begin{array}{c}\text { Isolated post- } \\
\text { capillary PH }\end{array}$ & $\begin{array}{c}\text { Combined pre-capillary } \\
\text { and post-capillary PH }\end{array}$ \\
\hline Mean pulmonary artery pressure & $<25 \mathrm{~mm} \mathrm{Hg}$ & $>25 \mathrm{~mm} \mathrm{Hg}$ & $\geq 25 \mathrm{~mm} \mathrm{Hg}$ & $>25 \mathrm{~mm} \mathrm{Hg}$ \\
Pulmonary capillary wedge pressure & $<15 \mathrm{~mm} \mathrm{Hg}$ & $<15 \mathrm{~mm} \mathrm{Hg}$ & $\geq 15 \mathrm{~mm} \mathrm{Hg}$ & $\geq 15 \mathrm{~mm} \mathrm{Hg}$ \\
Pulmonary vascular resistance & $<3 \mathrm{WU}$ & $\geq 3 \mathrm{WU}$ & $<3 \mathrm{WU}$ & $>3 \mathrm{WU}$ \\
\hline
\end{tabular}

$\mathrm{PH}$, pulmonary hypertension; WU, Woods Units.

worst prognosis. ${ }^{4}$ While pulmonary capillary wedge pressure and pulmonary artery pressure appear to be adverse prognostic markers in $\mathrm{CA}$, the prevalence of $\mathrm{PH}$ and its haemodynamic profiles in CA are currently unknown. ${ }^{56}$

As myocardial amyloid deposition results in a restrictive cardiomyopathy and left-sided heart failure, one might postulate IPCPH to be predominant. However, case series of precapillary $\mathrm{PH}$ in patients with CA suggest a more complex aetiology. ${ }^{7}$ Additionally, rates of CPCPH in patients with heart failure with preserved ejection fraction (HFpEF) and heart failure with reduced ejection fraction (HFrEF) are as high as $11 \%-40 \%$, suggesting that longstanding postcapillary PH can lead to pulmonary vascular remodelling and worse prognosis. ${ }^{48}$ Amyloid deposition within the pulmonary vasculature in CA may also accelerate pulmonary vascular disease irrespective of left heart pressures. $^{79}$

We performed a single-centre, retrospective analysis to identify the prevalence of $\mathrm{PH}$ and haemodynamic $\mathrm{PH}$ profiles among patients with CA. We also explored which haemodynamic and cardiovascular magnetic resonance (CMR) markers of pulmonary vascular disease were associated with adverse events in this population. We hypothesised that $\mathrm{PH}$ would be common among CA and that IPCPH would be the predominant phenotype. We further theorised that markers of right ventricular dysfunction and pulmonary vascular disease would be associated with worse outcomes in this population.

\section{METHODS}

We retrospectively identified consecutive patients with confirmed CA-either AL or ATTR-CA—who had undergone both comprehensive CMR examination and RHC between October 2010 and July 2019 at a single academic medical centre (figure 1). CA was defined in accordance with consensus guidelines as positive endomyocardial biopsy demonstrating amyloid fibrils, positive extracardiac biopsy with typical cardiac imaging features or grade $\geq 2$ uptake on technetium pyrophosphate scan. ${ }^{10} \mathrm{CA}$ subtype was determined either histologically or noninvasively by grade $\geq 2$ uptake on technetium pyrophosphate scan in the absence of a monoclonal light chain. ${ }^{10}$ Patients were excluded if RHC and CMR were performed $\geq 1$ year apart from each other or if there was insufficient chart documentation to confirm CA diagnosis and subtype. The Ohio State University Biomedical Sciences Institutional
Review Board approved this retrospective study and waived informed consent.

\section{Patient involvement}

As this study was retrospective, there was, therefore, no direct patient involvement in the design or analysis of this research.

\section{Clinical data}

Clinical characteristics and comorbidity information were obtained through a review of the electronic medical record at the time of CMR and included: age, gender, body mass index, CA subtype (immunoglobulin light chain or ATTR), haematocrit, troponin, B-type natriuretic peptide, history of hypertension, diabetes, hyperlipidaemia, heart failure and New York Heart Association class. All-cause mortality and combined major adverse cardiovascular events (MACE) including death plus heart failure hospitalisation-defined as admission for decompensated heart failure with evidence of congestion requiring intravenous loop diuretics-were adjudicated by chart review.

\section{Right heart catheterisation}

Haemodynamic parameters collected included right atrial, mean pulmonary artery, pulmonary artery

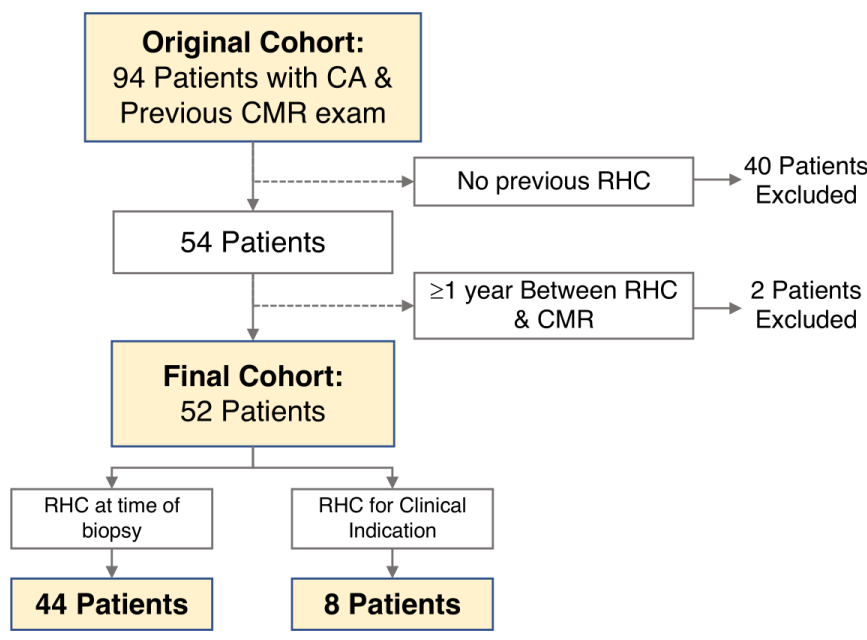

Figure 1 Study schematic. Figure depicting the schematic for inclusion and exclusion of patients. Patients with cardiac amyloidosis (CA) who had undergone cardiovascular magnetic resonance (CMR) and right heart catheterisation (RHC) within 1 year were included. Of the 94 patients who underwent CMR, 52 were ultimately included in the analysis. 
Table 2 Comparison of clinical parameters across groups

\begin{tabular}{|c|c|c|c|c|c|c|c|}
\hline & \multirow{2}{*}{$\begin{array}{l}\text { Entire cohort } \\
\mathrm{N}=52\end{array}$} & \multicolumn{4}{|l|}{$\mathrm{PH}$ group } & \multirow[b]{2}{*}{$P$ value } \\
\hline & & & $\begin{array}{l}\text { No PH } \\
(n=5)\end{array}$ & $\begin{array}{l}\text { Pre-capillary PH } \\
(n=3)\end{array}$ & $\begin{array}{l}\text { IPC-PH } \\
(\mathrm{n}=29)\end{array}$ & $\begin{array}{l}\text { CpcPH } \\
(n=15)\end{array}$ & \\
\hline \multicolumn{2}{|l|}{ Age (years) } & $69 \pm 9$ & $76 \pm 6$ & $68 \pm 8$ & $66 \pm 10$ & $71 \pm 8$ & 0.13 \\
\hline \multicolumn{2}{|c|}{ Gender $\mathrm{n}$ (\% male) } & $44(85 \%)$ & $4(80 \%)$ & $3(100 \%)$ & $26(89.7 \%)$ & $11(73.3 \%)$ & 0.45 \\
\hline \multirow[t]{3}{*}{ Ethnicity } & White & $34(65 \%)$ & $5(100 \%)$ & $0(0 \%)$ & $21(72.4 \%)$ & $8(53.3 \%)$ & 0.07 \\
\hline & Black & $17(33 \%)$ & $0(0 \%)$ & $3(100 \%)$ & $7(24.1 \%)$ & $7(46.7 \%)$ & \\
\hline & Other & $1(2 \%)$ & $0(0 \%)$ & $0(0 \%)$ & $1(3.4 \%)$ & $0(0 \%)$ & \\
\hline \multicolumn{2}{|l|}{ BMI $\left(\mathrm{kg} / \mathrm{m}^{2}\right)$} & $26.6(24.1-30.9)$ & $23.9(21.8-25.0)$ & $25.4(23.7-28.8)$ & $28.2(25.5-31.5)$ & $24.3(23.2-32.2)$ & 0.04 \\
\hline \multicolumn{2}{|c|}{ Amyloid subtype $n$ (\% ATTR) } & $31(60 \%)$ & $2(40 \%)$ & $3(100 \%)$ & $16(55.2 \%)$ & $10(66.7 \%)$ & 0.34 \\
\hline \multicolumn{2}{|c|}{ Hypertension n (\%) } & $27(52 \%)$ & $1(20 \%)$ & $2(66.7 \%)$ & $15(51.7 \%)$ & $9(60 \%)$ & 0.44 \\
\hline \multicolumn{2}{|c|}{ Hyperlipidaemia n (\%) } & $28(54 \%)$ & $1(20 \%)$ & $0(0 \%)$ & $18(62.1 \%)$ & $9(60 \%)$ & 0.08 \\
\hline \multicolumn{2}{|c|}{ Diabetes n (\%) } & $11(21 \%)$ & $0(0 \%)$ & $1(33.3 \%)$ & $6(20.7 \%)$ & $4(26.7 \%)$ & 0.60 \\
\hline \multicolumn{2}{|c|}{ Congestive heart failure $\mathrm{n}(\%)$} & $50(96 \%)$ & $4(80 \%)$ & $3(100 \%)$ & $29(100 \%)$ & $14(93.3 \%)$ & 0.16 \\
\hline \multirow[t]{4}{*}{ NYHA class } & In (\%) & $1(2 \%)$ & $1(20 \%)$ & $0(0 \%)$ & $0(0 \%)$ & $0(0 \%)$ & 0.13 \\
\hline & II n (\%) & $19(37 \%)$ & $3(60 \%)$ & $2(66.7 \%)$ & $10(34.5 \%)$ & $4(26.7 \%)$ & \\
\hline & III n (\%) & $24(46 \%)$ & $1(20 \%)$ & $1(33.3 \%)$ & $14(48.3 \%)$ & $8(53.3 \%)$ & \\
\hline & IV n (\%) & $8(15 \%)$ & $0(0 \%)$ & $0(0 \%)$ & $5(17.2 \%)$ & $3(20 \%)$ & \\
\hline \multicolumn{2}{|c|}{ Creatinine (mg/dL) } & $1.11(0.93-1.38)$ & $1.05(0.95-1.38)$ & $1.50(1.30-1.59)$ & $1.09(0.93-1.30)$ & $1.0(0.88-1.46)$ & 0.43 \\
\hline \multicolumn{2}{|c|}{ Haematocrit (\%) } & $38.2 \pm 5.0$ & $38.3 \pm 2.1$ & $31.0 \pm 3.9$ & $37.6 \pm 5.4$ & $40.0 \pm 3.9$ & 0.05 \\
\hline \multicolumn{2}{|l|}{ BNP (ng/L) } & $518(262-764)$ & $349(213-785)$ & $518(447-1017)$ & $520(240-706)$ & $552(304-849)$ & 0.84 \\
\hline \multicolumn{2}{|c|}{ Troponin (ng/mL) } & $0.18(0.08-0.27)$ & $0.04(0.04-0.40)$ & $0.16(0.09-0.18)$ & $0.22(0.08-0.30)$ & $0.18(0.12-0.23)$ & 0.41 \\
\hline \multicolumn{2}{|c|}{ Right atrial pressure $(\mathrm{mm} \mathrm{Hg})$} & $13.2 \pm 7.2$ & $3.6 \pm 2.6^{\star}$ & $13.3 \pm 7.2$ & $14.3 \pm 6.6^{\star} \dagger$ & $14.1 \pm 7.4^{\star} \dagger$ & 0.002 \\
\hline \multicolumn{2}{|c|}{ Mean PA pressure $(\mathrm{mm} \mathrm{Hg})$} & $30.0 \pm 6.0$ & $19.2 \pm 3.8^{\star}$ & $27.3 \pm 0.6$ & $29.6 \pm 4.0^{\star} \dagger$ & $35.0 \pm 5.1^{*} \dagger$ & $<0.001$ \\
\hline \multicolumn{2}{|c|}{$\begin{array}{l}\text { Pulmonary capillary wedge pressure } \\
(\mathrm{mm} \mathrm{Hg})\end{array}$} & $19.9 \pm 5.5$ & $9.4 \pm 3.0^{\star}$ & $13.7 \pm 0.6^{\star} \dagger$ & $21.9 \pm 4.3^{\star} \dagger$ & $20.8 \pm 3.8^{\star}$ & $<0.001$ \\
\hline \multicolumn{2}{|c|}{ fick cardiac output (I/min) } & $4.15 \pm 1.15$ & $3.77 \pm 1.12$ & $4.17 \pm 0.40$ & $4.54 \pm 1.15$ & $3.50 \pm 0.99$ & 0.08 \\
\hline \multicolumn{2}{|c|}{ Fick cardiac index $\left(\mathrm{L} / \mathrm{min} / \mathrm{m}^{2}\right)$} & $2.1(1.6-2.1)$ & $1.8(1.7-2.7)$ & $2.0(1.7-2.3)$ & $2.1(1.8-2.4)$ & $1.7(1.4-2.2)$ & 0.26 \\
\hline \multicolumn{2}{|c|}{ PVR (mm Hg/L/min) } & $2.6 \pm 1.2$ & $2.7 \pm 0.5^{\star}$ & $3.3 \pm 0.4$ & $1.7 \pm 0.6^{\star} \dagger$ & $4.1 \pm 0.8^{\star} \dagger$ & $<0.001$ \\
\hline \multicolumn{2}{|c|}{ TPG (mm Hg) } & $10.1 \pm 4.1$ & $9.8 \pm 2.4^{*}$ & $13.7 \pm 0.6$ & $7.6 \pm 2.5 \dagger$ & $14.2 \pm 3.7^{\star} \dagger$ & $<0.001$ \\
\hline \multicolumn{2}{|c|}{$\mathrm{DPG}(\mathrm{mm} \mathrm{Hg})$} & $1(0-3.8)$ & $3.0(0.8-4.3)$ & $6.0(5.0-6.8)$ & $0.0(0.0-0.3) \dagger$ & $3.0(1.0-6.3) \dagger$ & 0.003 \\
\hline
\end{tabular}

Intergroup p-values are provided in online supplemental table 1.

${ }^{*} \mathrm{P}<0.05$ between no $\mathrm{PH}$ group and depicted $\mathrm{PH}$ group.

$+\mathrm{P}<0.05$ between indicated $\mathrm{PH}$ groups.

ATTR, transthyretin; BMI, body mass index; BNP, B type natriuretic peptide; $\mathrm{CpcPH}$, combined post and pre-capillary PH; DPG, diastolic pressure gradient; IPC-PH, isolated post-capillary PH; NYHA, New York Heart Association; PA, pulmonary arterial; PCWP, pulmonary capillary wedge pressure; PH, pulmonary hypertension; PVR, pulmonary vascular resistance; TPG, transpulmonary gradient.

diastolic, pulmonary artery systolic and pulmonary capillary wedge pressures, Fick cardiac output and index and pulmonary vascular resistance (PVR). Transpulmonary gradient (TPG) was calculated as the difference between mean pulmonary artery and mean pulmonary capillary wedge pressure. Diastolic pulmonary gradient (DPG) was calculated as the difference between pulmonary artery diastolic and mean pulmonary capillary wedge pressures. ${ }^{11} 12$ Patients were categorised invasively as having no $\mathrm{PH}$, precapillary PH, IPCPH or CPCPH using previously described criteria (table 1 ). ${ }^{3}$ We used a mean pulmonary artery pressure cut-off of $\geq 25 \mathrm{~mm} \mathrm{Hg}$ instead of $20 \mathrm{~mm} \mathrm{Hg}$, as the study data predate the publication of recent European Respiratory Society (ERS) recommendations. ${ }^{13}$

\section{Cardiovascular magnetic resonance}

Patients underwent comprehensive CMR examinations with cine imaging, T1/T2 mapping and late gadolinium enhancement imaging using standard Society of Cardiovascular Magnetic Resonance guidelines. ${ }^{14}$ Atrial and ventricular volumes were indexed to body surface area. Global circumferential, radial and longitudinal left and right ventricular strain were assessed from the cine images using feature tracking available in CVI42 imaging software (CMR42, Circle Cardiovascular Imaging Calgary, Alberta, Canada). ${ }^{15}$

\section{Statistical analysis}

Statistical analyses were performed using MedCalc Statistical Software V.18.5 (MedCalc Software bvba, Ostend, Belgium; 


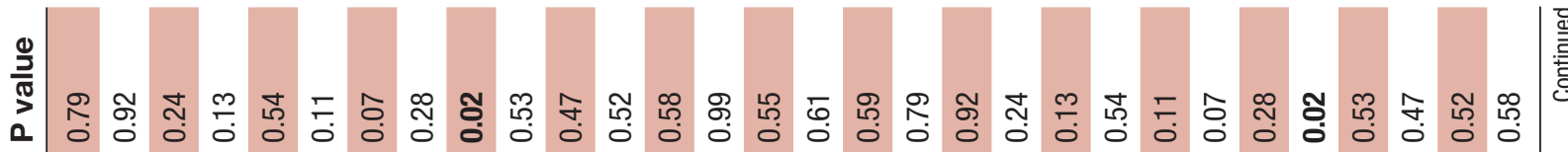
का

लं ॐ ळ

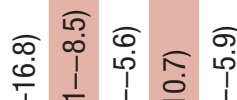
ळ क $\begin{gathered}\infty \\ \infty \\ \infty\end{gathered}$
m. 1.

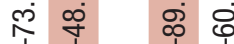
0 $I \quad \stackrel{0}{0}$ 过

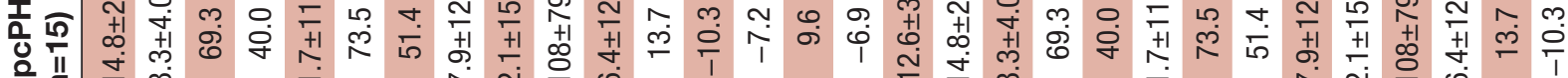

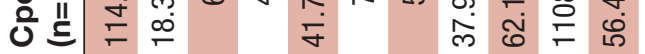

$$
\text { 守 }
$$$$
\text { ले ชิ }
$$

\section{Еิ}

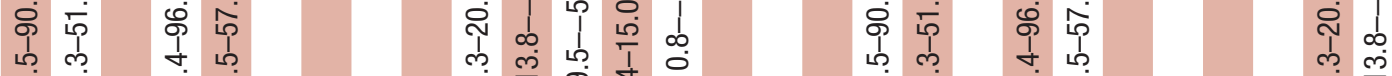

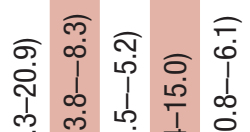

๘)

우

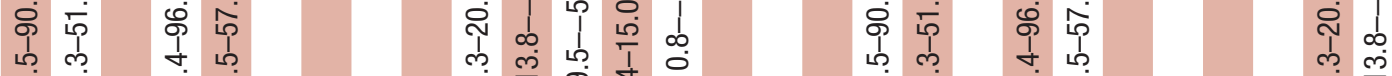

क़่

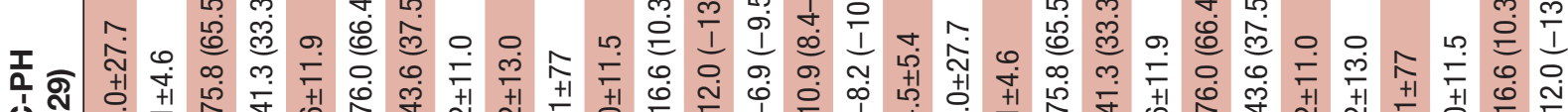

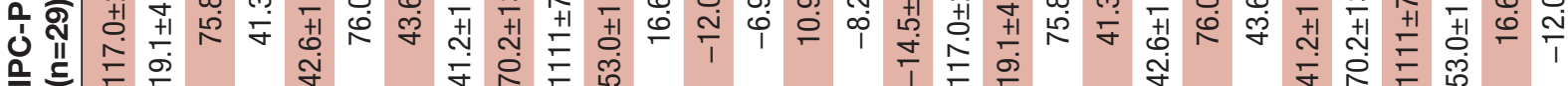

อิ

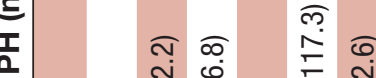

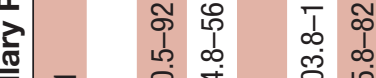

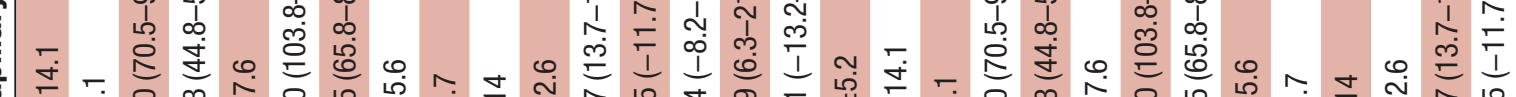

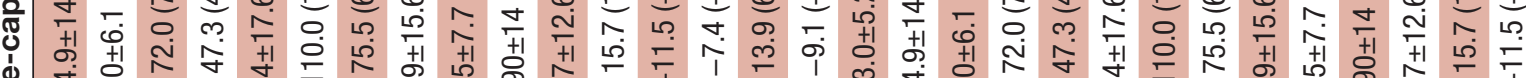

d.

a

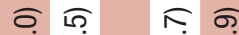

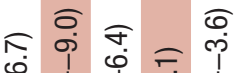

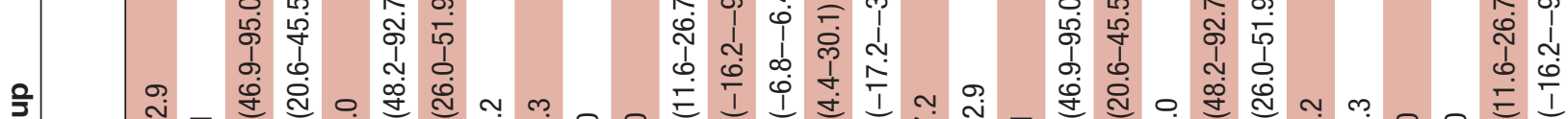

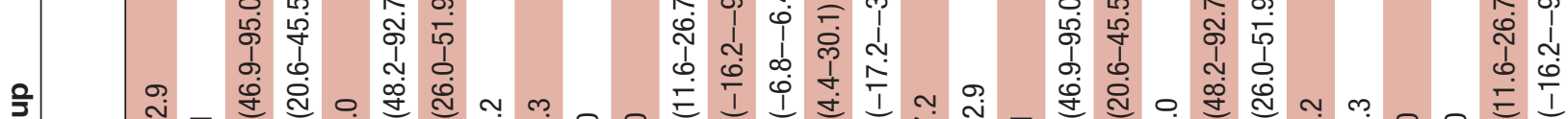

लिं

ले ळं

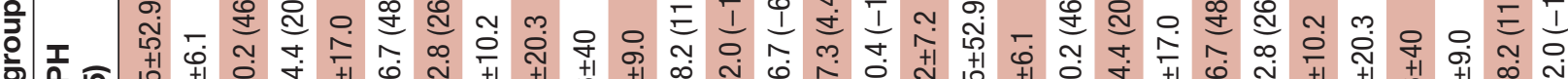

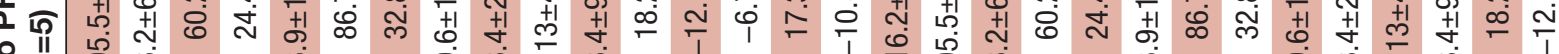

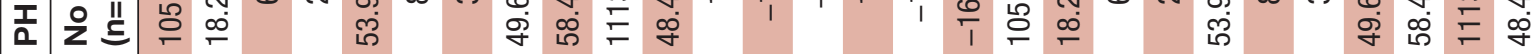

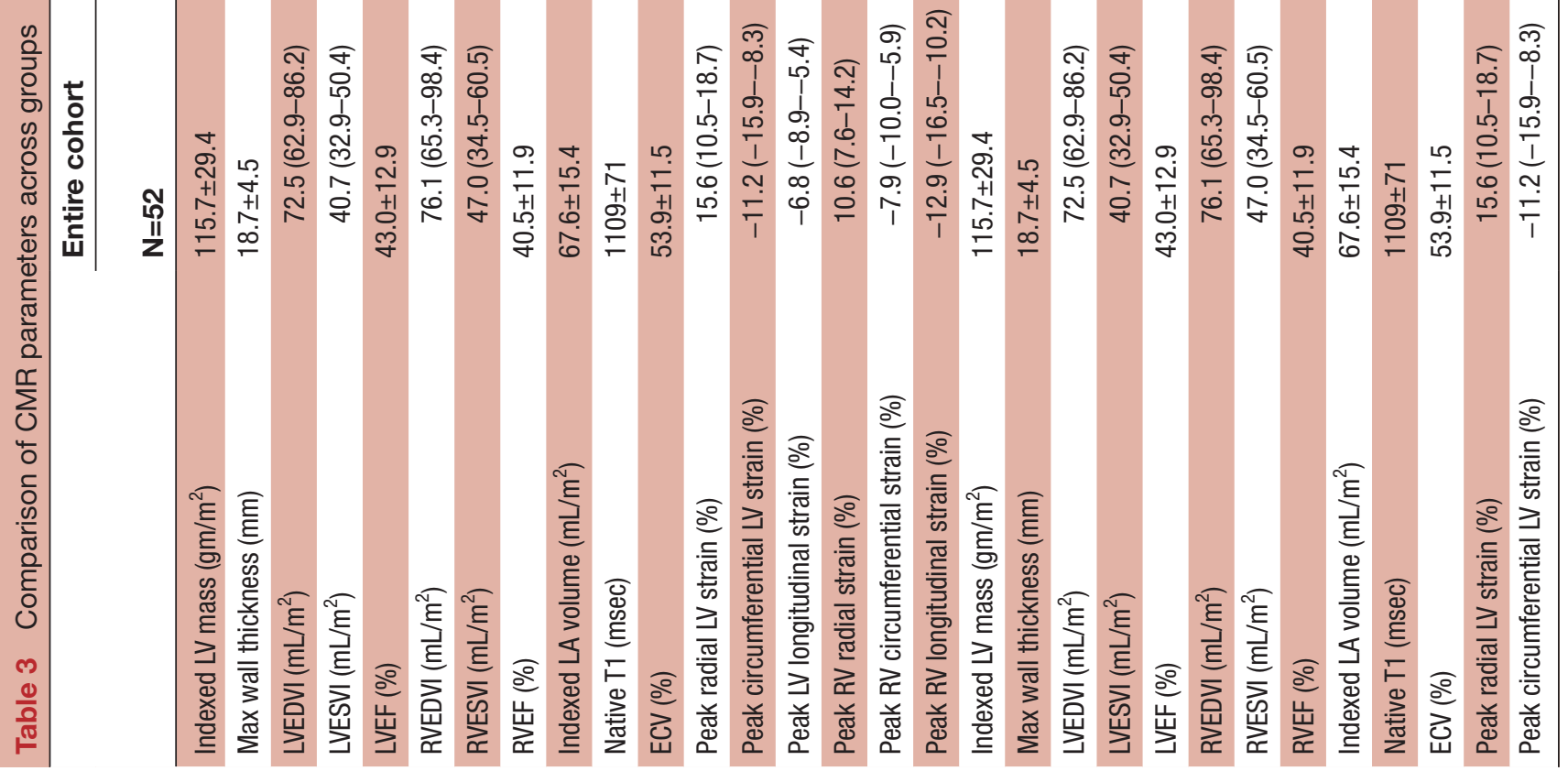


https://www.medcalc.org; 2018) and R (R Core Team (R: A language and environment for statistical computing. $\mathrm{R}$ Foundation for Statistical Computing, Vienna, Austria). Intergroup differences between haemodynamic $\mathrm{PH}$ profiles were assessed using one-way analysis of variance (ANOVA) for all continuous variables. Normal distribution of residuals was assessed using the Shapiro Wilk test and equality of error variances was determined using the Levene's test in the ANOVA analysis. The data are expressed as mean $\pm \mathrm{SD}$ and ANOVA $p$ value is reported for those variables where normality was not rejected and the Levene's test was not positive. If the Levene's test was positive, Welch's ANOVA was used. ${ }^{16}$ The non-parametric Kruskal-Wallis test was used when normality was rejected. ${ }^{17}$ The data are presented as median (IQR). Scheffe's and Dunn post hoc tests were used, respectively, for positive ANOVA and Kruskal-Wallis tests to perform pairwise comparison of the four groups. $\chi^{2}$ test was performed for all categorical variables. The data are presented as frequency with percentage. $\mathrm{p}<0.05$ was considered statistically significant for all tests.

The relationship between right ventricle (RV) function-as assessed by right ventricular ejection fraction (RVEF) and RV strain-was compared with extracellularvol (ECV), mean pulmonary artery and PVR using Pearson's correlation coefficient for normally distributed data and using Spearman's rank correlation coefficient for non-normal data. Regression diagnostics were performed to investigate the relationship between predictors and response variable and test model assumptions including linear relationship, no or little multicollinearity and homoscedasticity. Significant relationships were adjusted for age, CA subtype and $\mathrm{PH}$ phenotype using multivariable regression analysis. A log-rank test was used to determine if there were significant differences in the hospitalisation rate and survival distribution for the different $\mathrm{PH}$ phenotypes. Additionally, univariate Cox regression model was performed to investigate the independent predictors of the combined endpoint of death or heart failure hospitalisation and all-cause mortality. Proportionality assumptions of the Cox regression models were assessed by using Schoenfeld residuals. The deviance residuals and the dfbeta values were applied to examine influential observations. Martingale residuals against continuous predictors were used to assess nonlinearity and the functional form of predictors. HRs are presented as mean and 95\% CIs. Furthermore, to evaluate the importance of each parameter in predicting the combined endpoint of death or heart failure hospitalisation, random forest algorithm for survival analysis was applied. ${ }^{18}$ The authors agree to make all data available in a deidentified manner if requested.

\section{RESULTS}

\section{Prevalence of PH and haemodynamic profiles}

A total of 52 patients were included in the study (mean age $69 \pm 9$ years, $85 \%$ men, $35 \%$ non-white, $60 \%$ ATTRCA). RHC was performed for clinical purposes in eight 

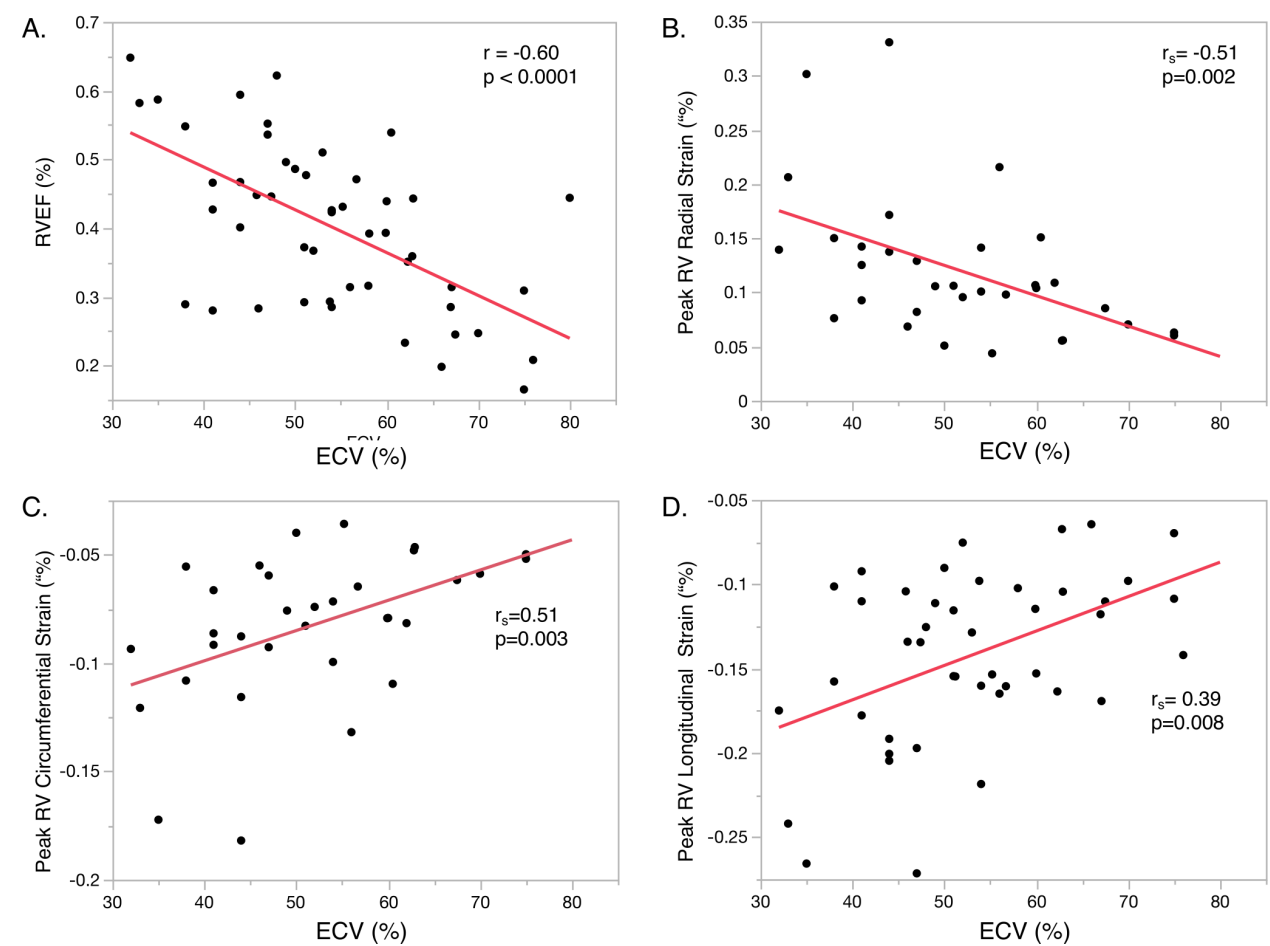

Figure 2 Correlation between right ventricular function and amyloid burden. This figure depicts the relationship between amyloid burden as assessed with extracellular volume (ECV) and cardiovascular magnetic resonance (CMR)derived measurements of right ventricular function. (A) right ventricular ejection fraction, peak right ventricular (B) radial, (C) circumferential and (D) longitudinal strain. Correlation was determined using Pearson's $(r)$ or Spearman's rank $\left(r_{s}\right)$ correlation coefficients.

patients $(15 \%)$ and at the time of endomyocardial biopsy in $44(85 \%)$ patients. The median time between RHC and CMR examination was 15 (4 to 42) days. Of these patients, $5(10 \%)$ had no $\mathrm{PH}, 3(6 \%)$ had isolated precapillary PH, 29 (55\%) had IPC-PH and 15 (29\%) had CPCPH. The prevalence of ATTR and AL subtype did not differ significantly among groups. There were no significant intergroup differences with respect to age, gender, ethnicity, comorbidities or New York Heart Association class (table 2). A comparison of haemodynamic data is presented in table 2; significant intergroup variables including p-values are presented in online supplemental table 1 . There were no significant intergroup differences in Fick Cardiac index.

\section{CMR characteristics}

With respect to CMR parameters, LV mass, indexed LV end diastolic volume, left ventricular ejection fraction (LVEF),
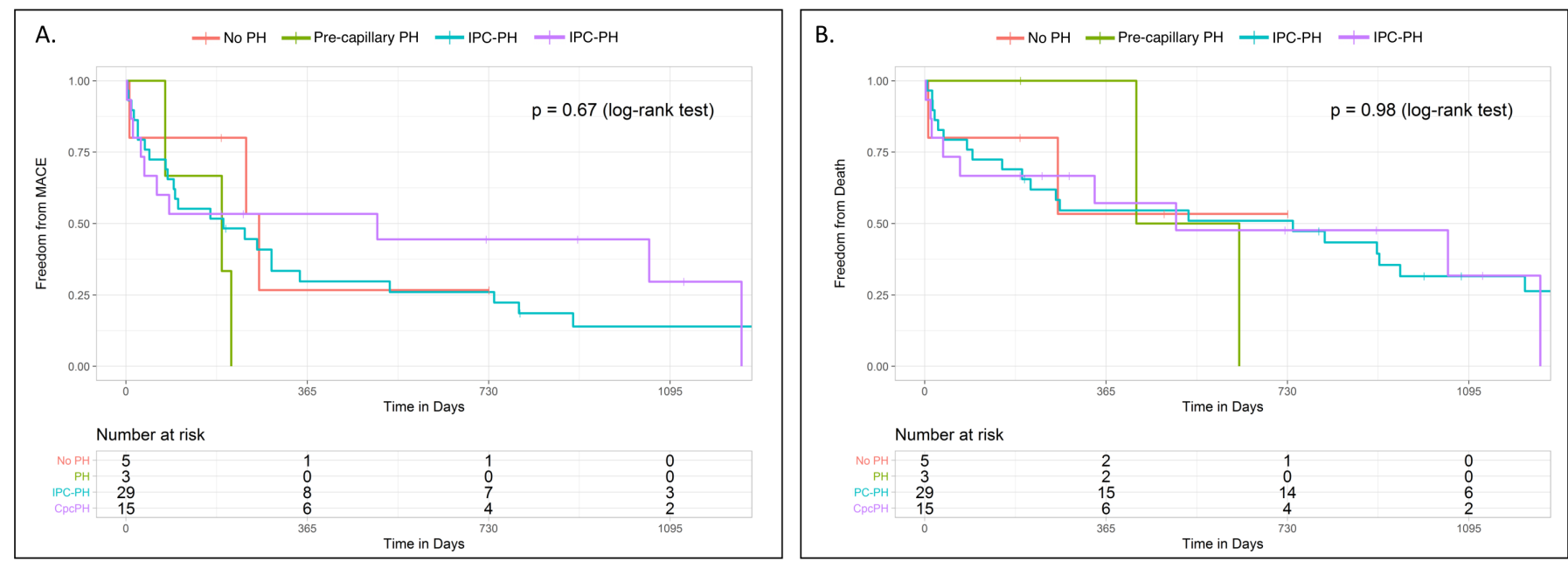

Figure 3 Major adverse events across haemodynamic PH profiles. This figure depicts Kaplan-Meier curves demonstrating time to (A) major adverse cardiovascular events - defined as death or heart failure hospitalisation-and (B) death among the four haemodynamic PH profiles. There were no significant differences in outcomes between groups using log-rank sum test ( $p$ values denoted in upper right hand corner). IPC, isolated postcapillary; PH, pulmonary hypertension. 
Table 4 Cox-regression analysis for predicting MACE and all-cause death

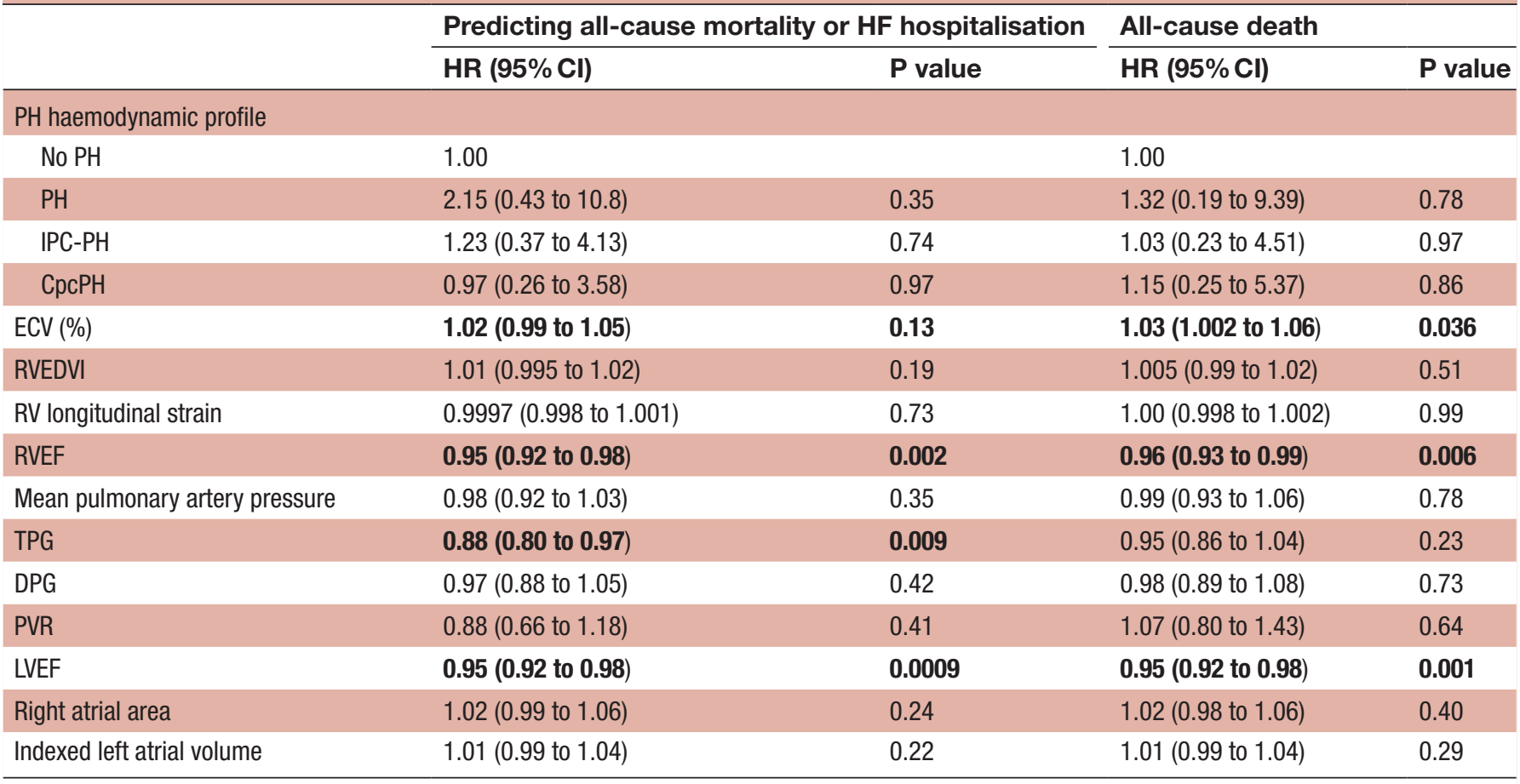

$\mathrm{CpcPH}$, Combined post and pre-capillary PH; DPG, diastolic pulmonary gradient; EDVI, indexed end diastolic volume; ICP-PH, isolated postcapillary PH; PH, pulmonary hypertension; PVR, pulmonary vascular resistance; RV, right ventricle; TPG, transpulmonary gradient.

RVEF, peak RV radial, circumferential and longitudinal strain did not differ significantly among PH groups (table 3 ). As compared with those without PH, LA volumes were significantly higher in those with IPCPH and CPCPH. There were no significant intergroup differences with respect to $\mathrm{ECV}$, native $\mathrm{T} 1$ relaxation time or LV strain. All patients had delayed enhancement of the LV and $39(80 \%)$ had RV involvement on late gadolinium enhancement imaging.

\section{Correlation between RV function, haemodynamics and amyloid burden}

There was a significant inverse correlation between RVEF and ECV $(r=-0.60, p<0.0001)$ (figure 2). Similarly, peak $R V$ radial $\left(r_{s}=-0.51, p=0.002\right)$, circumferential $\left(r_{s}=0.51\right.$, $\mathrm{p}=0.003)$ and longitudinal strain $\left(\mathrm{r}_{\mathrm{s}}=0.39, \mathrm{p}=0.008\right)$ were significantly associated with ECV. However, no significant correlation existed between ECV and mean pulmonary artery pressure $(p=0.38)$ or PVR $(p=0.23)$. Neither PVR nor mean pulmonary artery pressure was significantly associated with any of the RV strain parameters in univariate analysis. ECV also weakly correlated with LVEF $(r=-0.37$, $\mathrm{p}=0.009$ ). In multivariate analysis, ECV remained significantly associated with RVEF (t Ratio $=-3.31, \mathrm{p}=0.002$ ), peak radial ( $\mathrm{t}$-ratio $=-2.85, \mathrm{p}=0.009)$, and circumferential (t-ratio=2.58, $\mathrm{p}=0.016$ ) but not longitudinal RV strain $(\mathrm{p}=0.06)$ after adjusting for age, CA subtype, PH phenotype and Fick CI.

\section{Outcomes}

At a median follow-up of 194 days, 35 (67\%) patients had died and 27 (52\%) experienced heart failure hospitalisation. Combined MACE of death or heart failure hospitalisation occurred in $42(81 \%)$ of patients. The median time to first heart failure hospitalisation was 293 days (IQR, 104-1324 days) and median time to death was 317 days (IQR, 93-911 days).

There were no significant differences in either MACE or all-cause mortality among $\mathrm{PH}$ haemodynamic profiles in time to event analysis ( $\mathrm{p}=0.67$ by log-rank test, figure 3 ). Consistently, PH haemodynamic phenotypes were not significantly associated with all-cause mortality $(\mathrm{p}=0.98$ by log-rank test, figure 3 ).

In Cox regression analysis, RVEF (HR 0.95; 95\% CI 0.92 to $0.98 ; \mathrm{p}=0.002$ ), TPG (HR $0.88 ; 95 \%$ CI 0.80 to $0.97 ; \mathrm{p}=0.009$ ) and LVEF (HR $=0.95 ; 95 \%$ CI 0.92 to 0.98 , $\mathrm{p}=0.009 ; \mathrm{p}=0.0009)$ were all associated with combined MACE (table 4). Similarly, RVEF (HR 0.96; 95\% CI 0.93 to $0.99 ; \mathrm{p}=0.006)$ and LVEF $(\mathrm{HR}=0.95 ; 95 \% \mathrm{CI}, 0.92$ to $0.98, \mathrm{p}=0.009$ ) were independently associated with allcause mortality (table 4). ECV was significantly associated with mortality ( $\mathrm{HR}=1.03 ; 95 \% \mathrm{CI} 1.002$ to $1.06, \mathrm{p}=0.036$ ) but not MACE. Furthermore, LVEF, RVEF and TPG (sorted in the decreasing order of predictor importance) were significantly associated with MACE (figure 4). In contrast, indexed right ventricular end diastolic volume, peak right ventricular longitudinal strain, mean pulmonary artery pressure, PVR and DPG were not associated with death or MACE.

\section{DISCUSSION}

We evaluated the prevalence of $\mathrm{PH}$ and prognostic significance of right heart parameters in patients with CA. 


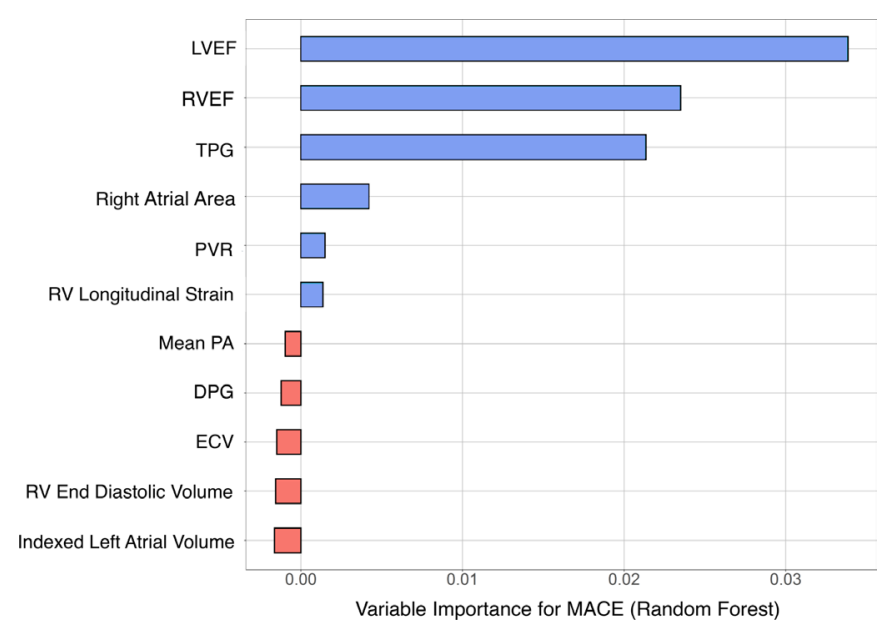

Figure 4 Importance of haemodynamic and cardiac imaging predictors of MACE: Importance of $\mathrm{RHC}$ and CMR imaging parameters in predicting the combined endpoint of death or heart failure hospitalisation. The relative importance was determined using Random Forest algorithm and is depicted in descending order of importance. Central Illustration: summary of key findings from our analysis. (A) $\mathrm{PH}$ is highly prevalent in cardiac amyloidosis (CA); while isolated post-capillary PH (IPC-PH) is most common, a substantial proportion of patients have combined post and pre-capillary $\mathrm{PH}$ (CPCPH). (B) Right ventricular dysfunction, as defined using RV ejection fraction and strain, correlates with amyloid burden as assessed by extracellular volume (ECV). (C) In Random Forest modelling, TPG, LVEF and RVEF were the most important variables in predicting major adverse cardiovascular events (MACE). CMR, cardiac magnetic resonance; DPG, diastolic pulmonary gradient; ECV, extracellular vol; EF, ejection fraction; LV, left ventricle; PA, pulmonary artery; PVR, pulmonary vascular resistance; $\mathrm{RHC}$, right heart catheterisation; $\mathrm{RV}$, right ventricle; TPG, transpulmonary gradient.

Four key points can be inferred from our analysis: (1) $\mathrm{PH}$ is highly prevalent at the time of CA diagnosis, (2) while isolated precapillary $\mathrm{PH}$ is rare, IPCPH and $\mathrm{CPCPH}$ occur frequently in CA, (3) right ventricular dysfunction correlates better with amyloid burden than with haemodynamic markers of pulmonary vascular disease and (4) TPG, right and left ventricular ejection fractions are associated with adverse outcomes in CA. The key findings of this manuscript are summarized in figure 5.

\section{Prevalence and hemodynamic profiles of PH in CA}

Our study highlights the prevalence and haemodynamic profile of $\mathrm{PH}$ in patients with $\mathrm{CA}$. $\mathrm{PH}$ was nearly ubiquitous in our cohort with over $88 \%$ of patients having mean pulmonary artery pressure $\geq 25 \mathrm{~mm} \mathrm{Hg}$. Additionally, $\mathrm{PH}$ was present at the time of diagnosis in $91 \%$ of cases, highlighting the need for earlier diagnosis of CA prior to onset of IPCPH and CPCPH. While we did not use a non-CA control group, these rates appear to be much higher than previous reported rates in the general HFpEF and HFrEF populations. ${ }^{8} 19$ Isolated precapillary $\mathrm{PH}$ was rare in our cohort, supporting the theory that pulmonary vascular disease rarely occurs in the absence of increased left-sided filling pressures. Yet, over onefourth of patients had haemodynamic profiles consistent with $\mathrm{CPCPH}$, suggesting that some element of intrinsic pulmonary vascular disease is not uncommon in CA. Pulmonary vascular disease is typically a late complication of left heart failure and is thought to occur due to the long-term effects of vascular distension and inflammation on the pulmonary vasculature. ${ }^{2021}$

The treatment of $\mathrm{PH}$ in the setting of left heart disease is complicated as excessive pulmonary vasodilation can worsen left-heart failure. However, despite these reasonable concerns, several recent pharmaceutical and interventional trials targeting the pulmonary vasculature have shown benefit in CPCPH. ${ }^{22-25}$ Whether targeted PH therapies may similarly benefit appropriately phenotyped patients with CA with CPCPH is currently unknown.

\section{Correlation of RV function with pulmonary vascular haemodynamics and amyloid burden}

Interestingly, contrary to our initial hypothesis, right ventricular function-as assessed using CMR — did not significantly differ between $\mathrm{PH}$ groups. Instead, right ventricular function correlated much more closely with amyloid burden as assessed by ECV than with haemodynamic $\mathrm{PH}$ profile. ECV has been previously shown to correlate with histologic amyloid burden. ${ }^{26} 27$

One potential explanation is that right ventricular dysfunction in CA may be driven by intrinsic right ventricular myopathy from amyloid deposition rather than from PH. Unlike other aetiologies of left heart failure, CA is an infiltrative cardiomyopathy, which frequently involves both ventricles. In previous biopsy and autopsy studies, amyloid deposits were identified in the RV in 85\%-95\% of patients with CA. ${ }^{28}$ Additionally, apical sparing of longitudinal strain-a classical finding in the LV-also occurs in the RV in CA. Both of these findings support the theory that amyloid infiltration of the RV directly contributes to right ventricular dysfunction in this population.

Pulmonary vascular disease, right heart failure, and outcomes Given the small size of our cohort and limited number of patients with precapillary $\mathrm{PH}$ and without $\mathrm{PH}$, our study was underperformed to detect differences in clinical outcomes across $\mathrm{PH}$ haemodynamic profiles. Additionally, the overall poor prognosis of the cohort further complicated our ability to detect intergroup differences in outcomes. Further studies-ideally in larger multicentre cohorts-are needed to determine whether $\mathrm{PH}$ haemodynamic profiles are prognostic in patients with CA.

We did find that TPG was associated with MACE in CA. Elevation in TPG is indicative of intrinsic pulmonary vascular disease and can differentiate CPCPH from PHLHD. ${ }^{12}$ Increased TPG has been previously associated with increased mortality in the HFpEF and HFrEF populations. ${ }^{11}$ Additionally, we validate previous studies in demonstrating an association between left and right ventricular function and adverse events in CA. ${ }^{30-33}$ 
Hemodynamic Profiles of Pulmonary Hypertension in Cardiac Amyloidosis

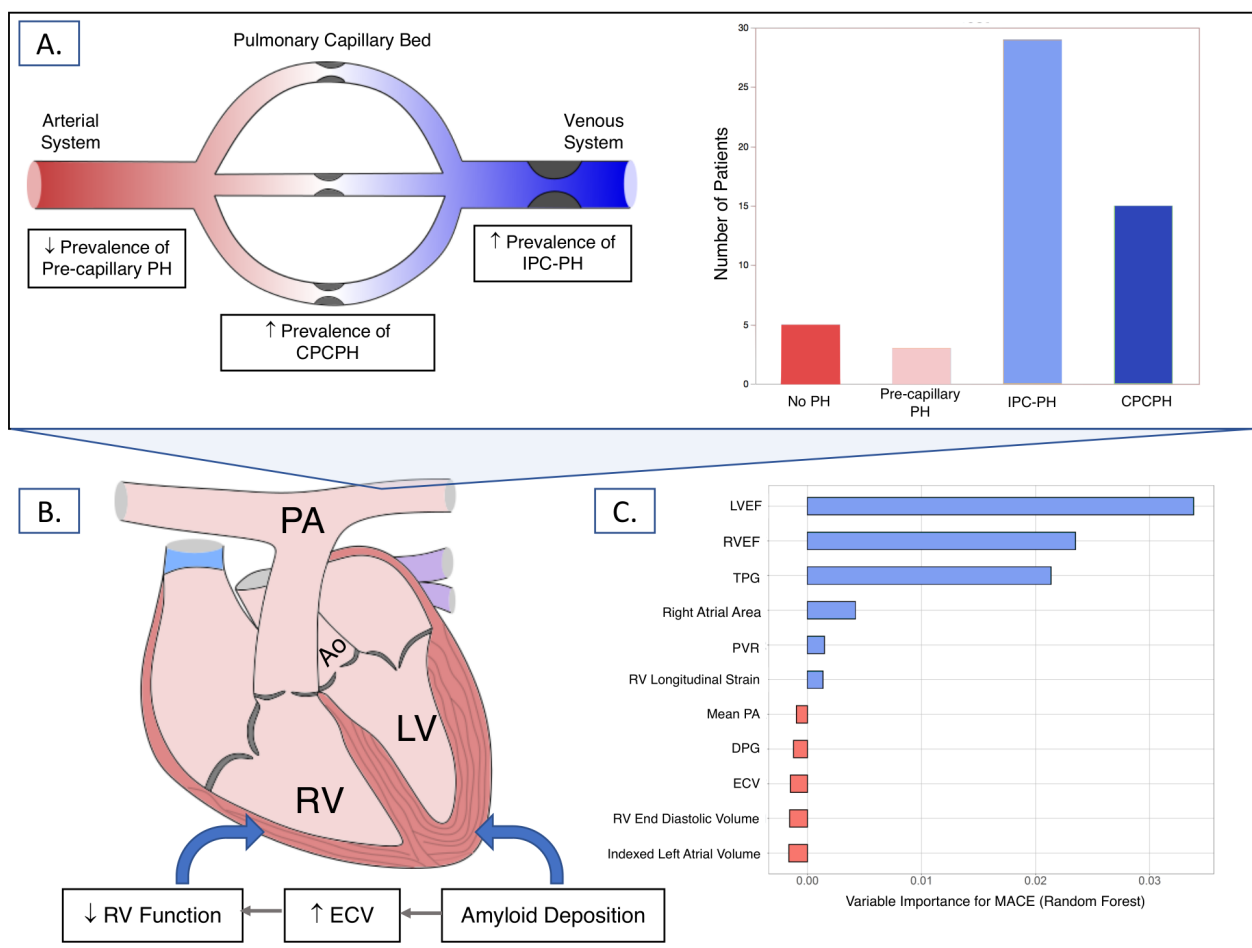

Figure 5 Summary of key findings from our analysis. (A) PH is highly prevalent in cardiac amyloidosis (CA); while isolated post-capillary $\mathrm{PH}$ (IPC-PH) is most common, a substantial proportion of patients have combined post and pre-capillary $\mathrm{PH}$ (CPCPH). (B) Right ventricular dysfunction, as defined using RV ejection fraction and strain, correlates with amyloid burden as assessed by extracellular volume (ECV). (C) In Random Forest modelling, TPG, LVEF and RVEF were the most important variables in predicting major adverse cardiovascular events (MACE). DPG, diastolic pulmonary gradient; ECV, extracellular vol; EF, ejection fraction; LV, left ventricle; PA, pulmonary artery; PH, pulmonary hypertension; PVR, pulmonary vascular resistance; $\mathrm{RV}$, right ventricle; TPG, transpulmonary gradient.

Given the small size of our cohort, we were unable to robustly control for covariates. Therefore, the relationships between these parameters and outcomes should be viewed as exploratory.

\section{Limitations}

Due to the small size of our cohort and the retrospective nature of the study, there exists the possibility of type II error due to confounding. With the size of our cohort, we additionally could not perform multivariable analysis to control for confounders due to model overfitting. ${ }^{345}$ Therefore, our findings should ideally be validated in larger multicentre cohorts, which would enable more robust controlling for covariates. Given its retrospective nature, RHC and CMR were not performed simultaneously. It is, therefore, possible that these studies may have been performed under different cardiac loading conditions. However, in the vast majority of patients, CMR and RHC were performed within 40 days of each other. Additionally, RHC and CMR were performed for clinical indications, which may select for sicker patients. However, as discussed above, a majority of RHCs were performed at the time of diagnosis during endomyocardial biopsy. Even after excluding RHC done for clinical indications, the prevalence of $\mathrm{PH}$ was $91 \%$, even higher than in the overall cohort. Our study also included both AL and ATTR cardiac amyloidosis, which may differ in their cardiac manifestations. While we were unable to further subdivide the cohort due to sample size, we did not note any significant differences in subtype prevalence among the haemodynamic profiles. Finally, based on survival data and CMR parameters, our cohort demonstrates advanced stage disease. It is unknown whether these results can be generalised to patients with CA with milder disease.

\section{CONCLUSION}

In conclusion, we demonstrate a high prevalence of $\mathrm{PH}$ among patients with CA even at the time of first diagnosis, highlighting a need for earlier diagnosis of CA. While IPCPH was the predominant haemodynamic phenotype identified, a substantial proportion of patients had CPCPH. RVEF and TPG were both associated with MACE in CA.

\section{Twitter Jeremy Slivnick @JSlivnickMD}

Contributors JS is the guarantor and accepts full responsibility for the work and/ or the conduct of the study. Dr. Slivnick had access to the data and controlled the decision to publish. All authors meet full criteria and requirements for authorship. The manuscript is not under consideration for publication elsewhere. All authors have read and approved the manuscript. This project did not receive funding from either grants or industry. None of the authors' disclosed entities directly supported or were involved in this project in any way.

Funding The authors have not declared a specific grant for this research from any funding agency in the public, commercial or not-for-profit sectors. 
Competing interests None declared.

Patient consent for publication Not applicable.

Ethics approval This study involves human participants and was approved by the Ohio State University institutional review board (IRB), study ID 2019H0293. This study was retrospectively performed through a review of the electronic health record (EHR) and cardiac magnetic resonance images. No patients were contacted and no diagnostic or therapeutic intervention was performed outside of routine clinical care. The Ohio State University institutional review board (IRB), therefore, agreed to waive informed consent for the purposes of this study.

Provenance and peer review Not commissioned; externally peer reviewed.

Data availability statement Data are available upon reasonable request. The authors of this manuscript have agreed to make the de-identified data available upon reasonable request.

Open access This is an open access article distributed in accordance with the Creative Commons Attribution Non Commercial (CC BY-NC 4.0) license, which permits others to distribute, remix, adapt, build upon this work non-commercially, and license their derivative works on different terms, provided the original work is properly cited, appropriate credit is given, any changes made indicated, and the use is non-commercial. See: http://creativecommons.org/licenses/by-nc/4.0/.

\section{ORCID iDs}

Jeremy Slivnick http://orcid.org/0000-0002-2327-4544

Juliet Varghese http://orcid.org/0000-0003-2882-2893

Saurabh Rajpal http://orcid.org/0000-0003-0772-8478

\section{REFERENCES}

1 Hahn VS, Yanek LR, Vaishnav J, et al. Endomyocardial Biopsy Characterization of Heart Failure With Preserved Ejection Fraction and Prevalence of Cardiac Amyloidosis. JACC Heart Fail 2020;8:712-24.

2 Castaño A, Narotsky DL, Hamid N, et al. Unveiling transthyretin cardiac amyloidosis and its predictors among elderly patients with severe aortic stenosis undergoing transcatheter aortic valve replacement. Eur Heart J 2017;38:2879-87.

3 Simonneau G, Montani D, Celermajer DS, et al. Haemodynamic definitions and updated clinical classification of pulmonary hypertension. Eur Respir J 2019;53. doi:10.1183/13993003.019132018. [Epub ahead of print: 2401 2019].

4 Vanderpool RR, Saul M, Nouraie M, et al. Association between hemodynamic markers of pulmonary hypertension and outcomes in heart failure with preserved ejection fraction. JAMA Cardiol 2018;3:298-306

5 Duca F, Snidat A, Binder C, et al. Hemodynamic profiles and their prognostic relevance in cardiac amyloidosis. J Clin Med 2020;9:1093.

6 Russo C, Green P, Maurer M. The prognostic significance of central hemodynamics in patients with cardiac amyloidosis. Amyloid 2013;20:199-203.

7 Dingli D, Utz JP, Gertz MA. Pulmonary hypertension in patients with amyloidosis. Chest 2001;120:1735-8.

8 Miller WL, Grill DE, Borlaug BA. Clinical features, hemodynamics, and outcomes of pulmonary hypertension due to chronic heart failure with reduced ejection fraction: pulmonary hypertension and heart failure. JACC Heart Fail 2013;1:290-9.

9 Smith RR, Hutchins GM, Moore GW, et al. Type and distribution of pulmonary parenchymal and vascular amyloid. Correlation with cardiac amyloid. Am J Med 1979;66:96-104.

10 Dorbala S, Ando Y, Bokhari S, et al. ASNC/AHA/ASE/EANM HFSA/ISA/SCMR/SNMMI expert consensus recommendations for multimodality imaging in cardiac amyloidosis: Part 1 of 2Evidence base and standardized methods of imaging. J Card Fail 2019;25:e1-39.

11 Gerges C, Gerges M, Lang MB. Diastolic pulmonary vascular pressure gradient: a predictor of prognosis in "out-of-proportion" pulmonary hypertension. Chest 2013;143:758-66.

12 Naeije R, Vachiery J-L, Yerly P, et al. The transpulmonary pressure gradient for the diagnosis of pulmonary vascular disease. Eur Respir J 2013;41:217-23.
13 Hoeper MM. The new definition of pulmonary hypertension. Eur Respir J 2009;34:790-1.

14 Kramer CM, Barkhausen J, Bucciarelli-Ducci C, et al. Standardized cardiovascular magnetic resonance imaging (CMR) protocols: 2020 update. J Cardiovasc Magn Reson 2020;22:17.

15 CVI42 user manual. Available: https://www.circlecvi.com/docs/ product-support/manuals/cvi42_user_manual_v5.5.pdf

16 Larson MG. Analysis of variance. Circulation 2008;117:115-21.

17 Bewick V, Cheek L, Ball J. Statistics review 10: further nonparametric methods. Crit Care 2004;8:196-9.

18 Huynh-Thu VA, Saeys Y, Wehenkel L, et al. Statistical interpretation of machine learning-based feature importance scores for biomarker discovery. Bioinformatics 2012;28:1766-74.

19 Tampakakis E, Leary PJ, Selby VN, et al. The diastolic pulmonary gradient does not predict survival in patients with pulmonary hypertension due to left heart disease. JACC Heart Fail 2015;3:9-16.

20 Fayyaz AU, Edwards WD, Maleszewski JJ, et al. Global pulmonary vascular remodeling in pulmonary hypertension associated with heart failure and preserved or reduced ejection fraction. Circulation 2018;137:1796-810.

21 Guazzi M, Ghio S, Adir Y. Pulmonary hypertension in HFpEF and HFrEF: JACC review topic of the week. J Am Coll Cardiol 2020;76:1102-11.

22 Zhang $\mathrm{H}$, Zhang J, Chen $\mathrm{M}$, et al. Pulmonary artery denervation significantly increases 6-min walk distance for patients with combined pre- and post-capillary pulmonary hypertension associated with left heart failure. JACC Cardiovasc Interv 2019;12:274-84.

23 Belyavskiy E, Ovchinnikov A, Potekhina A, et al. Phosphodiesterase 5 inhibitor sildenafil in patients with heart failure with preserved ejection fraction and combined pre- and postcapillary pulmonary hypertension: a randomized open-label pilot study. BMC Cardiovasc Disord 2020;20:408.

24 Hefke T, Zittermann A, Fuchs U, et al. Bosentan effects on hemodynamics and clinical outcome in heart failure patients with pulmonary hypertension awaiting cardiac transplantation. Thorac Cardiovasc Surg 2012;60:26-34.

25 Lewis GD, Shah R, Shahzad K, et al. Sildenafil improves exercise capacity and quality of life in patients with systolic heart failure and secondary pulmonary hypertension. Circulation 2007;116:1555-62.

26 Banypersad SM, Sado DM, Flett AS. Quantification of myocardial extracellular volume fraction in systemic $\mathrm{AL}$ amyloidosis: an equilibrium contrast cardiovascular magnetic resonance study. Circ Cardiovasc Imaging 2013;6:34-9

27 Duca F, Kammerlander AA, Panzenböck A, et al. Cardiac magnetic resonance $T$, mapping in cardiac amyloidosis. JACC Cardiovasc Imaging 2018;11:1924-6.

28 Pellikka PA, Holmes DR, Edwards WD. Endomyocardial biopsy in 30 patients with primary amyloidosis and suspected cardiac involvement. Arch Intern Med 1988;148:662-6.

29 Smith TJ, Kyle RA, Lie JT. Clinical significance of histopathologic patterns of cardiac amyloidosis. Mayo Clin Proc 1984;59:547-55.

30 Cappelli F, Porciani MC, Bergesio F, et al. Right ventricular function in $\mathrm{AL}$ amyloidosis: characteristics and prognostic implication. Eur Heart J Cardiovasc Imaging 2012;13:416-22.

31 Wan K, Sun J, Han Y, et al. Right ventricular involvement evaluated by cardiac magnetic resonance imaging predicts mortality in patients with light chain amyloidosis. Heart Vessels 2018;33:170-9.

32 Ghio S, Perlini S, Palladini G, et al. Importance of the echocardiographic evaluation of right ventricular function in patients with AL amyloidosis. Eur J Heart Fail 2007:9:808-13.

33 Bellavia D, Pellikka PA, Dispenzieri A, et al. Comparison of right ventricular longitudinal strain imaging, tricuspid annular plane systolic excursion, and cardiac biomarkers for early diagnosis of cardiac involvement and risk stratification in primary systematic (AL) amyloidosis: a 5-year cohort study. Eur Heart J Cardiovasc Imaging 2012;13:680-9.

34 Moons KGM, Altman DG, Reitsma JB, et al. Transparent reporting of a multivariable prediction model for individual prognosis or diagnosis (TRIPOD): explanation and elaboration. Ann Intern Med 2015;162:W1-73.

35 Concato J, Peduzzi P, Holford TR, et al. Importance of events per independent variable in proportional hazards analysis. I. Background, goals, and general strategy. J Clin Epidemiol 1995;48:1495-501. 